\title{
A NOTE ON AN INEQUALITY OF M. MARCUS AND M. NEWMAN
}

\section{HENRYK MINC ${ }^{1}$}

The permanent of an $n$-square matrix $A=\left(a_{i j}\right)$ is denoted by $p(A)$ and is defined by

$$
p(A)=\sum_{\sigma \in S_{n}} \prod_{i=1}^{n} a_{i \sigma(i)},
$$

where $S_{n}$ is the symmetric group of degree $n$. A real matrix $S$ is said to be doubly stochastic if its entries are nonnegative and its row sums and column sums are all 1 .

In a recent paper [2] Marcus and Newman proved that if $A$ is a symmetric positive semi-definite doubly stochastic matrix then

$$
p(A) \geqq n ! / n^{n}
$$

with equality if and only if $A=J_{n}$, the matrix all of whose entries are $1 / n$. This result is a partial answer to a conjecture of van der Waerden [3] stating that if $S$ is any doubly stochastic matrix then $p(S)$ $\geqq p\left(J_{n}\right)=n ! / n^{n}$ with equality if and only if $S=J_{n}$.

In the present note we extend the result of Marcus and Newman to all positive semi-definite hermitian matrices which have $e=(1,1, \cdots, 1)$ as a characteristic vector and prove it by a new method. We first establish a lemma which is a weakened version of Theorem 2 in [1] and use it in conjunction with the following inequality also due to Marcus and Newman (Theorem 5 in [2]).

If $A$ and $B$ are complex $n$-square matrices then

$$
|p(A B)|^{2} \leqq p\left(A A^{*}\right) p\left(B^{*} B\right)
$$

with equality if and only if either (a) a row of $A$ or a column of $B$ is zero, or (b) there exist a diagonal matrix $G$ and a permutation matrix $P$ such that $A^{*}=B G P$.

Let $\Phi_{n}$ denote the set of positive semi-definite hermitian $n$-square matrices which have $e$ as a characteristic vector. In other words, $\Phi_{n}$ contains all nonnegative multiples of positive semi-definite hermitian $n$-square matrices whose row sums are all 1 . Thus if $H \in \Phi_{n}$ then $H J_{n}=\lambda_{1} J_{n}$ for some nonnegative $\lambda_{1}$.

Presented to the Society, November 17, 1962; received by the editors September 17, 1962.

1 This research was supported by the Air Force Office of Scientific Research. 
Lemma. For any $H$ in $\Phi_{n}$ there exists a matrix $B$ in $\Phi_{n}$ such that $B^{2}=H$.

Proof. Let all the row sums of $H$ be equal to $\lambda_{1} \geqq 0$. Then $\lambda_{1}$ is a characteristic root of $H$ and $e$ is a corresponding characteristic vector. There exists therefore a unitary matrix $U$ with $e / \sqrt{ } n$ as its first column vector and such that

$$
U^{*} H U=\operatorname{diag}\left(\lambda_{1}, \lambda_{2}, \cdots, \lambda_{n}\right),
$$

where $\lambda_{1}, \lambda_{2}, \cdots, \lambda_{n}$ are the nonnegative characteristic roots of $H$. Let $D=\operatorname{diag}\left(\sqrt{\lambda_{1}}, \sqrt{\lambda_{2}}, \cdots, \sqrt{\lambda_{n}}\right)$ and let $B=U D U^{*}$. Clearly $B$ is positive semi-definite hermitian and $B^{2}=H$. It remains to prove that $e$ is a characteristic vector of $B$. Let $X^{(1)}$ denote the first column vector of a matrix $X$. Then

$$
U^{*} e=U^{*} \sqrt{ } n U^{(1)}=\sqrt{ } n\left(U^{*} U\right)^{(1)}=\left(\begin{array}{c}
\sqrt{ } n \\
0 \\
\vdots \\
0
\end{array}\right) .
$$

Hence $X U^{*} e=\sqrt{ } n X^{(1)}$ for any $n$-square matrix $X$. In particular

$$
B e=U D U^{*} e=\sqrt{ } n(U D)^{(1)}=\sqrt{ } n U D^{(1)}=\sqrt{ }\left(\lambda_{1} n\right) U^{(1)}=\sqrt{ } \lambda_{1} e .
$$

TheOREM. If $H \in \Phi_{n}$ and the row sums of $H$ are all equal to $\lambda_{1}$ then

$$
p(H) \geqq n !\left(\lambda_{1} / n\right)^{n}
$$

with equality if and only if either a row of $H$ is zero or $H$ is a nonnegative multiple of $J_{n}$.

Proof. By the lemma, $H=B^{2}$ where $B \in \Phi_{n}$ and therefore $B=B^{*}$ and $B J_{n}=\sqrt{ } \lambda_{1} J_{n}$. Hence, by (3),

$$
\left|p\left(B J_{n}\right)\right|^{2} \leqq p\left(B B^{*}\right) p\left(J_{n}^{*} J_{n}\right)
$$

i.e.,

$$
\left(p\left(\sqrt{ } \lambda_{1} J_{n}\right)\right)^{2} \leqq p(H) p\left(J_{n}\right)
$$

and therefore

$$
p(H) \geqq \lambda_{1}^{n} p\left(J_{n}\right)=n !\left(\lambda_{1} / n\right)^{n} .
$$

Equality will occur (see conditions for equality in (3)) if and only if either (a) a row of $B$, and therefore a row of $H$, is zero, or (b) there exist a diagonal matrix $G$ and a permutation matrix $P$ such that 
$B=J_{n} G P$. In case (b), $H=B B^{*}=J_{n} G^{2} J_{n}$ and clearly $J_{n} G^{2} J_{n}$ $=\left(\operatorname{tr}\left(G^{2}\right) / n\right) J_{n}$.

CoRollary. If $S$ is a symmetric positive semi-definite doubly stochastic n-square matrix then

$$
p(S) \geqq n ! / n^{n}
$$

with equality if and only if $S=J_{n}$.

\section{REFERENCES}

1. Marvin Marcus and Henryk Minc, Some results on doubly stochastic matrices, Proc. Amer. Math. Soc. 13 (1962), 571-579.

2. Marvin Marcus and Morris Newman, Inequalities for the permanent function, Ann. of Math. (2) 75 (1962), 47-62.

3. B. L. van der Waerden, Aufgabe 45, Jber. Deutsch. Math.-Verein. 35 (1926), 117.

UNIVERSITY OF FLORIDA 